Genentech, Lilly, Janssen, Array BioPharma, GSK, Morphotek, Pfizer, Advaxis, AstraZeneca, Immunogen, Regeneron, and Nucana.

Drs. Han, Bender, and Follana have nothing to disclose.

Drs. Bacque and Li are employees of GlaxoSmithKline.

\section{NON-SURGICAL MANAGEMENT OF MALIGNANT BOWEL OBSTRUCTION IN ADVANCED OVARIAN CANCER PATIENTS - A SYSTEMATIC REVIEW AND META- ANALYSIS}

${ }^{1}$ Praveena Idaikkadar, ${ }^{2}$ Athina Georgiou, ${ }^{1}$ Simon Skene, ${ }^{1}$ Agnieszka Michael. ${ }^{1}$ University of Surrey; ${ }^{2}$ Kings College London

\subsection{6/ijgc-2020-ESG0.115}

Introduction/Background Ovarian cancer is the most lethal gynaecological malignancy and the 6 th most common cancer among women globally. The incidence of malignant bowel obstruction (MBO) in patients with advanced disease is up to $51 \%$. It presents a very distressing scenario for patients, their families and clinicians.

Management of $\mathrm{MBO}$ can be divided into surgical and medical management. Surgical management can involve direct resection, bypass surgery or stoma formation. Medical management includes endoscopic procedures, nasogastric tubes for decompression, bowel rest, parenteral feeding and symptom control such as chemotherapy, steroids, antisecretory drugs, analgesia and anti-emetics.

The rationale in choosing between surgical or medical management strategies is not well defined. High perioperative morbidity (up to 90\%) and mortality (up to 40\%) can make surgery a risky choice and there is increasing evidence that non-surgical management can significantly improve symptoms and quality of life.

The objective of this study was to evaluate the outcomes of patients with advanced ovarian cancer who undergo nonsurgical management of malignant bowel obstruction and conduct a meta-analysis to estimate median survival.

Methodology A literature search was carried out using the Pubmed, Embase and Medline online libraries up until November 2019. We also searched abstracts of scientific meetings, reference lists of included studies and contacted experts in the field. Relevant studies that met the inclusion criteria were independently selected by two of the co-authors and the data extracted and analysed separately.

Results In total 24 studies were found to be relevant for the systematic review and 9 met the eligibility criteria for the meta-analysis, a total of 2236 patients were included. Median survival for patients managed medically for bowel obstruction was 44 days (95\% CI 38-49 days, $\mathrm{I}^{2}=0 \%, \mathrm{P}$ $=0.128$ ).

Conclusion The quality of the included studies was relatively low, however the evidence shows that non-surgical management of bowel obstruction in advanced ovarian cancer patients results in a short survival period, but with controlled symptoms. Where quality of life is the main concern, this may be a feasible and effective strategy.

Disclosures Praveena Idaikkadar, Athina Georgiou and Simon Skene have no disclosures.

Agnieszka Michael has the following disclosures:

BMS, CLOVIS, ESAI, GSK, Ipsen, Novartis, Pfizer, Tesaro

\section{LEAP-005: EVALUATING THE SAFETY AND EFFICACY OF LENVATINIB AND PEMBROLIZUMAB IN PATIENTS PREVIOUSLY TREATED FOR OVARIAN CANCER, A MULTI-COHORT PHASE 2 STUDY}

${ }^{1}$ Antonio Gonzalez-Martin, ${ }^{2}$ Hyun Cheol Chung, ${ }^{3}$ Esma Saada-Bouzid, ${ }^{4}$ Eduardo Yanez, ${ }^{5}$ Hélène Senellart, ${ }^{6}$ Philippe A Cassier, ${ }^{7}$ Bristi Basu, ${ }^{8}$ Razi Ghori, ${ }^{9}$ Peter Kubiak, ${ }^{9}$ Alan Smith, ${ }^{8}$ Kevin Norwood, ${ }^{10}$ Zarnie Lwin. ${ }^{1}$ Clínica Universidad de Navarra; ${ }^{2}$ Yonsei Cancer Center, Yonsei University College of Medicine; ${ }^{3}$ Department of Medical Oncology, Centre de Lutte Contre Le Cancer Antoine Lacassagne; ${ }^{4}$ Oncology-Hematology Unit, Department of Internal Medicine, School of Medicine, Universidad de la Frontera; ${ }^{5}$ nstitut de Cancérologie de L'ouest, Centre René Gauducheau Ico; ${ }^{6}$ Department of Medical Oncology, Centre Léon Bérard; 'Department of Oncology, University of Cambridge; ${ }^{8}$ Merck and Co., Inc; ${ }^{9}$ Eisai Ltd., Hatfield, UK; ${ }^{10}$ Royal Brisbane and Women's Hospital, University of Queensland

\subsection{6/ijgc-2020-ESG0.116}

Introduction/Background Lenvatinib, an antiangiogenic multiple receptor tyrosine kinase inhibitor, plus pembrolizumab, a programmed death-1 immune checkpoint inhibitor, demonstrated promising clinical benefit in a previous phase $\mathrm{Ib} / \mathrm{II}$ trial across several cancer types (ClinicalTrials.gov, NCT02501096). We assessed clinical outcomes with lenvatinib plus pembrolizumab in patients with ovarian cancer in the ongoing, open-label, multicohort, phase II LEAP-005 study (ClinicalTrials.gov, NCT03797326).

\begin{tabular}{|c|c|c|c|}
\hline Studies & Est & imate $195 \%$ & $\because$ C.I.) \\
\hline Gadducci 1998 & 45.000 & $(30.300$, & $59.700)$ \\
\hline Bryan 2006 & 454.000 & (75.727, & $832.273)$ \\
\hline Mangili 1996 & 37.000 & $(23.045$, & $50.955)$ \\
\hline Matulonis 2005 & 89.000 & (14.913, & $163.087)$ \\
\hline Rath 2013 & 46.000 & (36.984, & 55.01 \\
\hline Pothuri 2004 & 56.000 & $(42.280$ & 69.72 \\
\hline Suidan 2017 & 36.000 & $(24.044$, & 47.95 \\
\hline Redman 1988 & 30.000 & $(-37.423$ & 97.42 \\
\hline DeEulis 2015 & 28.000 & $(-11 \cdot 395$ & 67.39 \\
\hline Overall $\left(I^{\wedge} 2=0 \%, P=0.128\right)$ & 43.965 & (38.652, & 49.27 \\
\hline
\end{tabular}

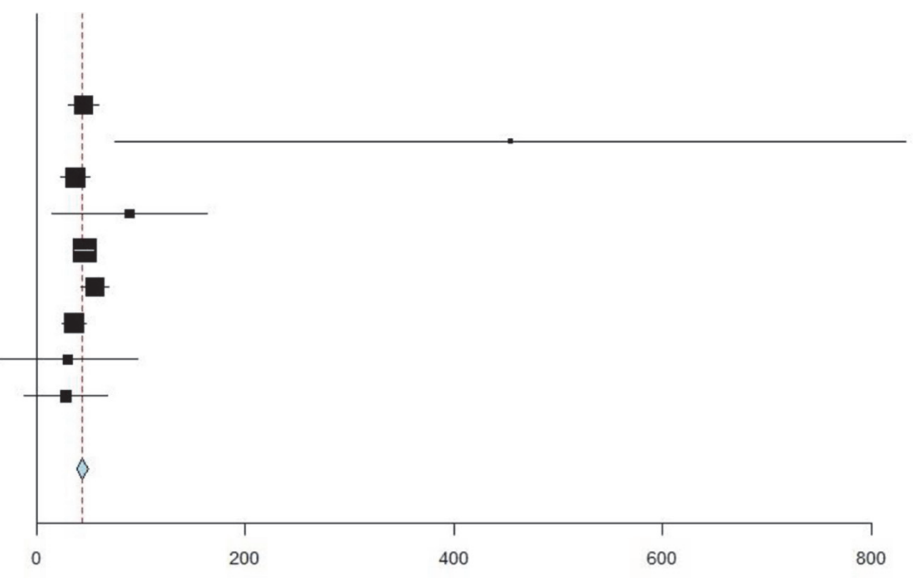


Methodology Female patients aged $\geq 18$ years with histologically/cytologically confirmed metastatic/unresectable ovarian cancer, measurable disease per Response Evaluation Criteria in Solid Tumors (RECIST v1.1), Eastern Cooperative Oncology Group (ECOG) performance status $0 / 1$, and 3 prior lines of therapy were enrolled. Patients received lenvatinib $20 \mathrm{mg}$ daily plus pembrolizumab $200 \mathrm{mg}$ every 3 weeks for 35 cycles, or until confirmed disease progression or unacceptable toxicity. Primary endpoints were objective response rate (ORR; response assessed every 9 weeks for 54 weeks, then every 12 weeks, by blinded independent central review per RECIST v1.1) and safety. Secondary endpoints included disease control rate, duration of response, and progression-free survival.

Results 31 patients with ovarian cancer received $\geq 1$ dose of lenvatinib plus pembrolizumab in LEAP-005 (median age 62 years [range 40-76]); median study follow-up was 7.8 months (range, 4.6-12.4) as of April 10, 2020. ORR was 32\% (95\% confidence interval, 17-51); other efficacy endpoints were also favorable (table 1). Treatment-related adverse events occurred in 29 (94\%) patients (table 1).

Conclusion Lenvatinib plus pembrolizumab demonstrated encouraging efficacy and manageable safety in patients with heavily pretreated ovarian cancer, including those with prior platinum failure and those with previous bevacizumab exposure.

Disclosures Antonio González-Martín: Advisory/Consultancy: Amgen, AstraZeneca, Clovis Oncology, Genmab, GSK, ImmunoGen, Mersana, Merck Sharp \& Dohme, Novartis, Oncoinvent, Pfizer/Merck, PharmaMar, Roche, Sotio; Speaker Bureau: AstraZeneca, PharmaMar, Roche, GSK; Research Grant/Funding: Roche, TESARO: A GSK Company; Travel/Accommodation/Expenses: AstraZeneca, PharmaMar, Roche, TESARO: A GSK Company

Hyun Cheol Chung: Grants/Research Support: Lilly, GSK, MSD, Merck-Serono, BMS/Ono, Taiho, Amgen, Beigene, Incyte; Honoraria: Merck-Serono, Lilly/Foundation Medicine; Consultation: Taiho, Celltrion, MSD, Lilly, Quintiles, BMS, Merck-Serono, Gloria, Beigene, Amgen, Zymework

\begin{tabular}{|c|c|c|}
\hline & & $\begin{array}{c}\text { Lenvatinib }+ \\
\text { Pembrolizumab }(n=31)\end{array}$ \\
\hline \multicolumn{3}{|l|}{ Efficacy } \\
\hline \multicolumn{2}{|c|}{ Confirmed ORR, \% $(95 \% \mathrm{CI})$} & $32(17-51)$ \\
\hline \multicolumn{2}{|c|}{ Disease control rate, ${ }^{a} \%(95 \% \mathrm{CI})$} & $74(55-88)$ \\
\hline \multicolumn{2}{|c|}{ Duration of response, median (range) months } & $\mathrm{NR}(1.5+$ to $7.9+)$ \\
\hline \multicolumn{2}{|c|}{ Progression-free survival, median $(95 \% \mathrm{CI})$ months } & $4.4(4.0-8.5)$ \\
\hline \multicolumn{3}{|c|}{ Responders by prior therapy, $\mathrm{n}(\%)^{\mathrm{b}}$} \\
\hline \multicolumn{2}{|c|}{ Platinum refractory/resistant $(\mathrm{n}=\mathbf{2 5})$} & $6(24)$ \\
\hline \multicolumn{2}{|c|}{ Bevacizumab exposed ( $\mathbf{n}=19)$} & $4(21)$ \\
\hline \multicolumn{2}{|c|}{ Treatment-related AEs, n (\%) } & $29(94)$ \\
\hline \multicolumn{2}{|c|}{ Grade 3-5 treatment-related AEs } & $21(68)$ \\
\hline \multicolumn{2}{|c|}{ Treatment-related AEs leading to death } & $1(3)^{\mathrm{c}}$ \\
\hline \multicolumn{2}{|c|}{ Treatment-related AEs resulting in treatment discontinuation } & $4(13)$ \\
\hline \multicolumn{3}{|c|}{ Treatment-related AEs occurring in $\geq 10$ patients } \\
\hline & Any Grade ${ }^{d}$ & Grade 3 \\
\hline Hypertension & $17(55)$ & $6(19)$ \\
\hline Fatigue & $13(42)$ & $3(10)$ \\
\hline Hypothyroidism & $13(42)$ & 0 \\
\hline Decreased appetite & $12(39)$ & 0 \\
\hline Diarrhoea & $12(39)$ & $1(3)$ \\
\hline Proteinuria & $10(32)$ & $2(6)$ \\
\hline \multicolumn{3}{|c|}{ 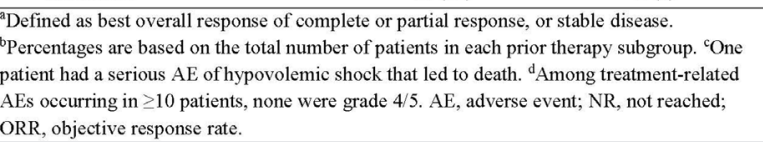 } \\
\hline
\end{tabular}

Esma Saada-Bouzid: Advisory/Consultancy: BMS, MSD; Travel/Accommodation: AstraZeneca, BMS, MSD

Eduardo Yanez: Consultant/Advisory Board: BMS, Merck Serono; Research Grant: Amgen, Pfizer, Astellas, BMS, Roche, Abbvie, MSD

Hélène Senellart: Nothing to disclose.

Philippe A. Cassier: Honoraria: Novartis, Roche/Genentech, Blueprint Medicines, Amgen, AstraZeneca; Research Funding: Novartis, Roche/Genentech, Lilly, Blueprint Medicines, Bayer, AstraZeneca, Celgene, Plexxikon, Abbvie, BMS, Merck Serono, MSD, Taiho Pharmaceutical, Toray Industries, Transgene, Loxo, GSK, Innate Pharma, Janssen (all paid to institution); Travel, Accomodations, Expenses: Roche, Amgen, Novartis, BMS, MSD, Netris Pharma.

Bristi Basu: Consultancy: GenMab (paid to institution); Advisory Board: Roche, Eisai Europe Limited, research grant from Celgene Ltd (all paid to institution); Speakers Bureau: Eisai Europe Ltd (paid to institution); Travel and Registration for Congress: Bayer.

Razi Ghori: employee of Merck Sharp \& Dohme Corp., a subsidiary of Merck \& Co., Inc., Kenilworth, NJ, USA

Peter Kubiak: employee of Eisai Inc., Woodcliff Lake, NJ, USA

Alan Smith: employee of Eisai Ltd., Hatfield, UK

Kevin Norwood: employee of Merck Sharp \& Dohme Corp., a subsidiary of Merck \& Co., Inc., Kenilworth, NJ, USA

Zarnie Lwin: Received honoraria for consulting, advisory role or travel sponsorship from AbbVie; AstraZeneca; BMS; Roche; and Merck

\section{THE EVOLVING ROLE OF PARP INHIBITORS IN NEWLY DIAGNOSED ADVANCED OVARIAN CANCER: THE EFFECT OF ONLINE EDUCATION ON CLINICIAN KNOWLEDGE, COMPETENCE AND CONFIDENCE}

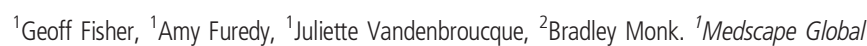
Education, New York, United States; ${ }^{2}$ University of Arizona College of Medicine, Phoenix, United States

\subsection{6/ijgc-2020-ESG0.117}

Introduction/Background The treatment of newly diagnosed advanced ovarian cancer is changing rapidly with the expanded use of PARP inhibitors. This study determined whether online continuing medical education could improve the knowledge, competence and confidence of oncologists and obstetricians/gynaecologists (obs/gyns) with regard to the application of PARP inhibitors in this setting.

Methodology A 30-minute online video panel discussion with synchronized slides was launched for physicians outside the USA in November 2019. Data was collected to January 2020. Educational effect was assessed with repeated-pairs pre-/post-activity, where individual participants served as their own control. 3 multiple-choice, knowledge questions and 1 self-efficacy, 5-point Likert scale confidence question were analyzed. Chi-squared test assessed pre- to post-activity change $(5 \%$ significance level, $\mathrm{P}<.05)$. Magnitude of change in total number of correct responses overall, and for each question, were determined with Cramer's V $(<.06=$ Modest,$\quad 0.06-0.15=$ Noticeable, $.16-.26=$ Consider able, $>.26=$ Extensive).

Results 157 oncologists and 152 obs/gyns completed pre- and post-activity questions. A positive educational effect was 